\title{
Carotid Artery Stiffness Accurately Predicts White Matter Hyperintensity Volume 20 Years Later: A Secondary Analysis of the Atherosclerosis Risk in the Community Study
}

\author{
(D) A. de Havenon, DK.-H. Wong, (D)A. Elkhetali, (D).S. McNally, (D).J. Majersik, and (D) N.S. Rost
}

\begin{abstract}
BACKGROUND AND PURPOSE: Arterial stiffness is a biomarker of cerebrovascular disease and dementia risk. Studies have shown an association between carotid artery stiffness and increased white matter hyperintensity volume and, as a result, reduced total brain volume on MR imaging, but none have had prolonged follow-up to fully evaluate the slow change seen in white matter hyperintensity volume and total brain volume with time. Our objective was to determine whether common carotid artery stiffness on sonography accurately predicts white matter hyperintensity volume and total brain volume on MR imaging more than 20 years later.
\end{abstract}

MATERIALS AND METHODS: We performed a secondary analysis of the Atherosclerosis Risk in the Community study to compare 5 measurements of carotid artery stiffness, including strain, distensibility, compliance, Stiffness index, and pressure-strain elastic modulus, with the white matter hyperintensity volume and total brain volume on a follow-up MR imaging using linear regression.

RESULTS: We included 1402 patients enrolled in the Atherosclerosis Risk in the Community study. There was a significant relationship between increasing carotid artery stiffness and both higher white matter hyperintensity volume and lower total brain volume on MR imaging, measured at a mean of 21.5 years later. In multivariable linear regression models, the carotid strain, distensibility, Stiffness index, and pressure-strain elastic modulus were associated with white matter hyperintensity volume. Only compliance was associated with total brain volume in the multivariate models.

CONCLUSIONS: Sonography measurements of carotid artery stiffness are predictive of white matter hyperintensity volume and total brain volume on MR imaging more than 20 years later. The association is more robust for white matter hyperintensity volume than total brain volume. These findings support the role of arterial stiffness as a method for identifying patients at risk of developing white matter hyperintensity volume and as a potential mechanism leading to small-artery disease of the brain.

ABBREVIATIONS: $\mathrm{CS}=$ carotid artery stiffness; $\mathrm{TBV}=$ total brain volume; $\mathrm{WMH}=$ white matter hyperintensity; $\mathrm{WMHv}=$ white matter hyperintensity volume

W hite matter hyperintensity (WMH), a radiographic signature of diffuse small cerebral vasculopathy, and brain atrophy on MR imaging are associated with impaired cognitive function and poor neurologic outcomes. ${ }^{1-3}$ Arterial stiffness is a marker of cerebrovascular disease, which is caused by advanced

\footnotetext{
Received December 27, 2018; accepted after revision May 28, 2019.

From the Department of Neurology (A.d.H., K.-H.W., A.E., J.S.M., J.J.M.), University of Utah, Salt Lake City, Utah; and Harvard Medical School (N.S.R.). Boston, Massachusetts.

Research reported in this publication was supported by an American Heart Association Scientist Development Grant 17SDG33670114 (Dr de Havenon).

The content of this work is solely the responsibility of the authors and does not represent the official views of the American Heart Association.

Please address correspondence to Adam de Havenon, MD, University of Utah, Department of Neurology, 175 N Medical Dr, Salt Lake City, UT 84132; e-mail: adam.dehavenon@hsc.utah.edu

http://dx.doi.org/10.3174/ajnr.A6115
}

age, hypertension, smoking, and diabetes. ${ }^{4,5}$ Carotid artery stiffness (CS), measured with sonography, is an independent risk factor for cerebrovascular disease, cognitive impairment, and mortality. ${ }^{4,6}$ Carotid wall stiffness is postulated to transmit the increase in blood pressure during systole to the brain, where it can harm the sensitive microvasculature, whose dysfunction is implicated in the pathogenesis of WMH and, as a result, brain atrophy. ${ }^{7}$ Most studies that examined the association of CS with WMH or brain atrophy have been cross-sectional with concurrent sonography and brain MR imaging or relied on aortic or peripheral arterial stiffness measurements rather than those of the carotid artery. ${ }^{4,8,9}$ Two longitudinal studies failed to show an association between CS and WMH or total brain volume (TBV) with time, but both had significantly shorter periods of follow-up. ${ }^{10,11}$ Given the slow pace of WMH and brain atrophy development, we sought to explore their association with CS during a more physiologically plausible time interval spanning 2 decades. 
Table 1: Baseline demographics at visit 2 and $P$ values for association with concurrent carotid distensibility measurement $^{a}$

\begin{tabular}{|c|c|c|}
\hline Variable & $\begin{array}{l}\text { Full Cohort } \\
(n=1402)\end{array}$ & $\begin{array}{c}P \text { Value for } \\
\text { Association with } \\
\text { Carotid Distensibility }\end{array}$ \\
\hline Age (mean) & $55.6 \pm 5.3$ & $<.001^{\mathrm{d}}$ \\
\hline Male (No.) (\%) & $571,40.7$ & .214 \\
\hline Caucasian (No.) (\%) & $1,023,73.0$ & $.007^{\mathrm{d}}$ \\
\hline Hypertension (No.) (\%) $(n=1396)$ & $280,20.1$ & $<.001^{\mathrm{d}}$ \\
\hline Diabetes (No.) (\%) $(n=1398)$ & $71,5.1$ & .265 \\
\hline History of myocardial infarction (No.) (\%) & $26,1.9$ & .898 \\
\hline History of stroke (No.) (\%) & $10,0.7$ & .602 \\
\hline Prevalent coronary heart disease (No.) (\%) & $16,1.2$ & .297 \\
\hline Current cigarette smoking (No.) (\%) $(n=1400)$ & $206,14.7$ & $<.001^{\mathrm{d}}$ \\
\hline Current alcohol consumption (No.) (\%) $(n=1400)$ & $846,60.4$ & $.009^{d}$ \\
\hline Body mass index (mean) & $27.1 \pm 4.6$ & $<.001^{\mathrm{d}}$ \\
\hline LDL cholesterol (mean) (mg/dL) $(n=1376)$ & $131.2 \pm 35.0$ & .493 \\
\hline HDL cholesterol (mean) (mg/dL) $(n=1328)$ & $49.3 \pm 16.7$ & .067 \\
\hline Fasting blood glucose (mean) (mmol/L) & $5.9 \pm 1.6$ & .077 \\
\hline $\begin{array}{l}\text { Antihypertensive in last } 2 \text { weeks (No.) (\%) } \\
(n=1397)\end{array}$ & $248,17.8$ & $<.001^{d}$ \\
\hline Aspirin in last 2 weeks (No.) (\%) $(n=1400)$ & $632,45.1$ & .369 \\
\hline Statin in last 2 weeks (No.) $(\%)(n=1400)$ & $29,2.1$ & 651 \\
\hline $\begin{array}{l}\text { Plaque in either carotid on ultrasound (No.) (\%) } \\
(n=1392)\end{array}$ & $310,22.3$ & .270 \\
\hline $\begin{array}{l}\text { Voltage of left ventricular leads on ECG (mean) } \\
(n=1391)\end{array}$ & $1174.2 \pm 525.4$ & $<.001^{\mathrm{d}}$ \\
\hline WMH volume (mean) (mL) & $17.5 \pm 17.4$ & \\
\hline Total brain volume (mean) $(\mathrm{mL})(n=1397)^{\mathrm{b}}$ & $1015.8 \pm 109.4$ & \\
\hline Ratio of WMH/TBV (mean) $(\%)(n=1397)^{b}$ & $1.7 \pm 1.7$ & \\
\hline Mean systolic blood pressure $(\mathrm{mm} \mathrm{Hg})^{c}$ & $135.0 \pm 12.4$ & \\
\hline Mean systolic SD $(\mathrm{mm} \mathrm{Hg})^{c}$ & $15.8 \pm 4.2$ & \\
\hline Mean number of blood pressure readings ${ }^{c}$ & $23.3 \pm 3.1$ & \\
\hline Years between carotid ultrasound and MRI (mean) & $21.5 \pm 0.9$ & \\
\hline
\end{tabular}

Note:-ECG indicates electrocardiography; HDL, high-density lipoprotein; LDL, low-density lipoprotein.

${ }^{a}$ Association tested with linear regression for continuous variables and Student $t$ test for categoric variables.

${ }^{b}$ Derived from visit $5 \mathrm{MRI}$.

c Derived from all available blood pressure readings from visit 2 through visit 5 .

d Significant.

\section{MATERIALS AND METHODS}

This is a secondary analysis of the Atherosclerosis Risk in the Community (ARIC) study, ${ }^{12}$ a large prospective epidemiologic study conducted in 4 communities in the United States. Participants were 45-64 years of age at enrollment in 1987-1989, and they continue to be followed periodically for vascular health status. No study-based intervention was provided. With a local institutional review board waiver from the University of Utah, we obtained the anonymized ARIC dataset from the National Heart, Lung, and Blood Institute Biologic Specimen and Data Repository Information Coordinating Center. We included patients who had both a carotid sonography at visit $2(1990-1992)$ and a brain MR imaging at visit 5 (2011-2013). No brain MR imaging was performed at visit 2 . The methods of carotid sonography measurements have been previously described, ${ }^{13}$ as have the methods of the white matter hyperintensity volume (WMHv) and TBV measurement on MR imaging. ${ }^{14}$ In short, the MR imaging was performed at a 3T field strength with a standardized protocol for all study sites. The ARIC MR imaging reading center (Mayo Clinic, Rochester, Minnesota) was responsible for quality control and interpretation of the scans. TBV was measured on a sagittal T1weighted 3D volumetric MPRAGE pulse sequence using FreeSurfer (Version 5.1; (http://surfer.nmr.mgh.harvard.edu) and an
ARIC-specific algorithm. WMHv was measured using similar methodology on an axial T2 FLAIR pulse sequence.

We included 5 measures of CS of the common carotid artery, which take into account the peak systolic diameter (PSD) of the carotid artery, end diastolic diameter (EDD), systolic blood pressure (SBP), diastolic blood pressure (DBP), and pulse pressure (PP). The 5 CS measures are the following: strain [(PSDEDD) / EDD], distensibility [100 $\times$ $\left.\left(\mathrm{PSD}^{2}-\mathrm{EDD}^{2}\right) /\left(\mathrm{PP} \times \mathrm{EDD}^{2}\right)\right]$, compliance $\left[\pi \times\left(\mathrm{PSD}^{2}-\mathrm{EDD}^{2}\right) /(4 \times\right.$ $\mathrm{PP})]$, Stiffness index $[\log (\mathrm{SBP} / \mathrm{DBP}) /$ strain], and pressure-strain elastic modulus [PP / strain]. For strain, distensibility, and compliance, higher values reflect less CS. For the Stiffness index and pressure-strain elastic modulus, higher values reflect more CS. All measures of CS were treated as continuous variables in statistical analyses, unless otherwise specified.

We report the association between visit 2 baseline demographics and carotid distensibility as a surrogate for CS, using linear regression and the Student $t$ test. We further divided the 5 CS measurements into quintiles and tested for differences in the mean $\mathrm{WMHv}$ and TBV with ANOVA. We fit multivariate linear regression models to the outcomes of WMHv and TBV. Model 1 was adjusted for all available systolic blood pressure measurements between visits 2 and 5 because chronic hypertension has shown the best correlation with WMHv. For the subsequent models, we tested candidate covariates from the demographic data in Table 1 with stepwise backward selection fit to the outcomes and set to a threshold of a $P<0.1$ for inclusion. Using this methodology, we adjusted model 2 for selected visit 2 demographics (the WMH model adjusted for patient age, sex, body mass index, high-density lipoprotein cholesterol level, current smoking, history of hypertension; the TBV model adjusted for patient age, sex, black race, fasting glucose level, and current smoking), and model 3 was adjusted for selected visit 3 demographics (1993-1995) (the WMH model adjusted for patient age, current cigarette smoking, history of hypertension, and voltage of left ventricular leads on electrocardiography; the TBV model adjusted for patient age, sex, black race, diabetes, and high-density lipoprotein cholesterol level). Sensitivity analyses were conducted adjusted for demographics from visit 4 and with the WMHv standardized to TBV (WMHv:TBV).

\section{RESULTS}

The final cohort included 1402 patients with a mean of 21.5 years between carotid sonography and MR imaging. The mean age at 
WMH (mean in $\mathrm{mL}$ )
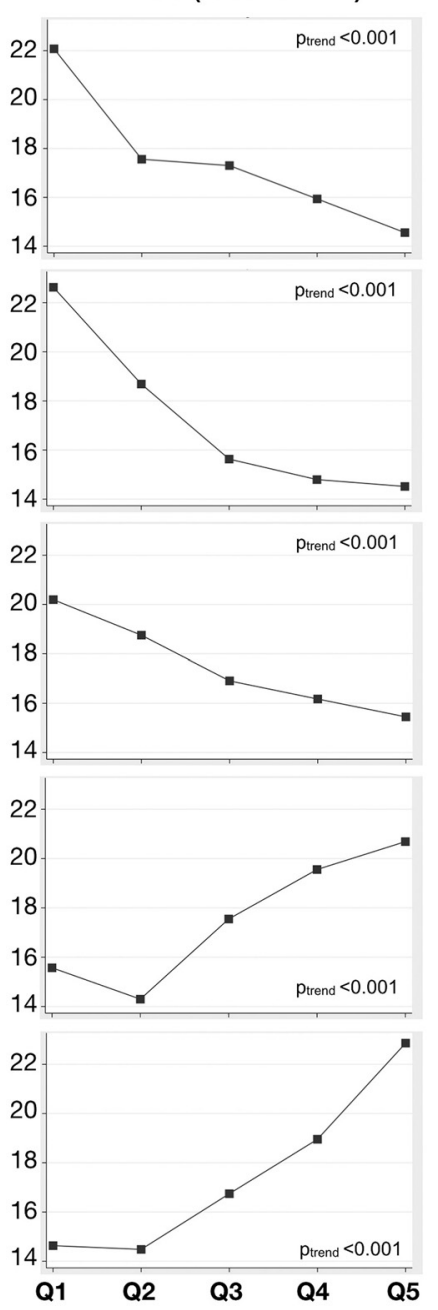

TBV (mean in $\mathrm{mL}$ )

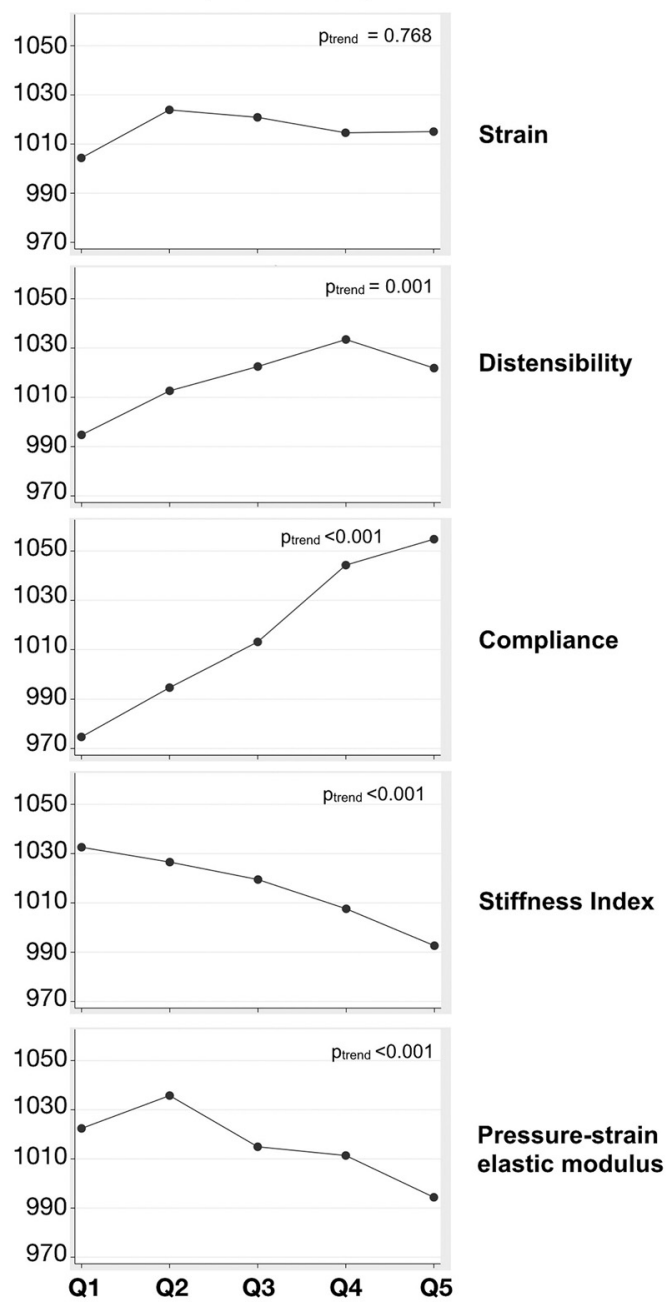

FIGURE. Mean values of WMH and TBV in the quintiles of carotid sonography measurements. For strain, distensibility, and compliance, the higher quintiles reflect less carotid stiffness. For the Stiffness index and pressure-strain elastic modulus, the higher quintiles reflect more carotid stiffness.

sonography was 57 years, and at MR imaging, it was 76 years. Baseline demographics from visit 2, when the carotid sonography was performed, are shown in Table 1. Although cerebrovascular risk factors were modestly prevalent (hypertension $=20.1 \%$, smoking $=14.7 \%$, diabetes $=5.1 \%$, plaque in either carotid artery on sonography $=22.3 \%$ ), only $0.7 \%$ had a history of prior stroke. The mean values for CS were the following: strain = $5.4 \% \pm 1.9 \%$, distensibility $=1.8 \% \pm 0.8 \% / \mathrm{kPa}$, compliance $=$ $8.0 \pm 3.1 \mathrm{~mm}^{3} / \mathrm{kPa}$, Stiffness index $=0.11 \pm 0.04$ (unitless), pressure-strain elastic modulus $=131.1 \pm 56.1 \mathrm{kPa}$. We found that the following visit 2 variables were significantly associated with increased CS: advanced patient age, black race, hypertension, smoking, not consuming alcohol, higher body weight, and evidence of left ventricular hypertrophy (LVH) on electrocardiography (Table 1).

The mean WMHv increased linearly with higher CS (Figure). In the linear regression models fit to $\mathrm{WMHv}$, carotid strain, distensibility, Stiffness index, and the pressure-strain elastic modulus were consistently associated with $\mathrm{WMHv}$, while compliance failed to maintain significance (Table 2). The relationship be- tween CS and TBV was less consistent than WMHv, and strain was not significantly predictive. Only compliance had an association with TBV in the multivariate models. These results were not changed in our sensitivity analyses (data not shown).

\section{DISCUSSION}

CS is associated with WMHv, and, to a lesser extent, with TBV on MR imaging obtained $>2$ decades later. The association with WMHv is more robust than for TBV, which is a multifactorial outcome that combines vascular injury and neurodegeneration. The percentage difference in WMHv between the least stiff to most stiff quintile of CS was on the order of $30 \%$, while for TBV, it was on the order of $2 \%$. These represent clinically meaningful differences that may translate to divergent outcome rates. Arterial stiffness has a direct pathophysiologic link to $\mathrm{WMH}$, which is caused by dysfunction of cerebral microvasculature that is hypothesized to be uniquely sensitive to the hypertension transmitted by stiffer arteries. ${ }^{7}$ In addition, our findings may be explained by shared long-term WMHv and CS risk-factor exposure, including hypertension, diabetes, smoking, and hyperlipidemia.

Our study is consistent with prior studies that were either cross-sectional or used peripheral measures of arterial stiffness, ${ }^{4,8,9}$ but the findings conflict with 2 prior longitudinal studies. The Second Manifestations of ARTerial disease-MR (SMART-MR) study looked at the change in WMHv and TBV and failed to find an association with CS, but it had only 4 years of follow-up. ${ }^{10}$ Likewise, in the Framingham Offspring Cohort, the carotidfemoral pulse wave velocity was not associated with change in WMHv and TBV, but the follow-up period was 6 years. ${ }^{11}$ While baseline MR imaging scans are not available for this analysis to account for temporal change in WMHv or TBV, these patients were relatively young at baseline, making the large volumes of WMH and brain atrophy seen on MR imaging at visit 5 , when the mean age was 76 years, an effective, though imperfect, proxy for change in WMHv or TBV. We posit that our findings reflect the more biologically meaningful interval between the measurements of CS and WMHv or TBV, which would allow the gradual progression of disease and accumulation of damage from increased arterial stiffness.

This analysis has the unique strength of a prolonged exposure period, a larger cohort than prior longitudinal studies, and validated measurements of the exposures and outcomes in a landmark epidemiologic study. Limitations include the lack of a base- 
Table 2: Linear regression fit to WMHv and TBV on the visit 5 MRI

\begin{tabular}{|c|c|c|c|}
\hline Ultrasound Variable & Model $1^{\mathrm{a}}$ & Model $2^{b}$ & Model $3^{c}$ \\
\hline \multicolumn{4}{|l|}{ WMHv (mL) } \\
\hline \multicolumn{4}{|l|}{ Strain } \\
\hline$\beta$ & -1.2 & -0.7 & -0.9 \\
\hline $95 \% \mathrm{Cl}$ & $(-1.7$ to -0.8$)$ & $(-1.2$ to -0.2$)$ & $(-1.4$ to -0.4$)$ \\
\hline$P$ value & $<.001$ & .007 & .001 \\
\hline \multicolumn{4}{|l|}{ Distensibility } \\
\hline$\beta$ & -1.9 & -1.6 & -1.7 \\
\hline $95 \% \mathrm{Cl}$ & $(-3.2$ to -0.6$)$ & $(-2.9$ to -0.3$)$ & $(-3.0$ to -0.4$)$ \\
\hline$P$ value & .003 & .019 & .009 \\
\hline \multicolumn{4}{|l|}{ Compliance } \\
\hline$\beta$ & -0.1 & -0.3 & -0.2 \\
\hline $95 \% \mathrm{Cl}$ & $(-0.4-0.2)$ & $(-0.6-0.1)$ & $(-0.5-0.1)$ \\
\hline$P$ value & .355 & .115 & .127 \\
\hline \multicolumn{4}{|l|}{ Stiffness index } \\
\hline$\beta$ & 45.1 & 30.6 & 35.9 \\
\hline $95 \% \mathrm{Cl}$ & $(23.1-67.2)$ & $(6.6-54.6)$ & $(12.5-59.3)$ \\
\hline$P$ value & $<.001$ & .012 & .003 \\
\hline \multicolumn{4}{|c|}{ Pressure-strain elastic modulus } \\
\hline$\beta$ & 0.03 & 0.03 & 0.04 \\
\hline $95 \% \mathrm{Cl}$ & $(0.02-0.05)$ & $(0.02-0.05)$ & $(0.02-0.05)$ \\
\hline$P$ value & .001 & $<.001$ & $<.001$ \\
\hline \multicolumn{4}{|l|}{$\mathrm{TBV}(\mathrm{mL})$} \\
\hline \multicolumn{4}{|l|}{ Strain } \\
\hline$\beta$ & 0.1 & 0.7 & 0.3 \\
\hline $95 \% \mathrm{Cl}$ & $(-2.9-3.2)$ & $(-1.7-3.1)$ & $(-2.2-2.8)$ \\
\hline$P$ value & .940 & .581 & .820 \\
\hline \multicolumn{4}{|l|}{ Distensibility } \\
\hline$\beta$ & 4.1 & 4.4 & 2.8 \\
\hline $95 \% \mathrm{Cl}$ & $(-4.0-12.3)$ & $(-1.7-10.4)$ & $(-3.4-9.0)$ \\
\hline$P$ value & .319 & .158 & .382 \\
\hline \multicolumn{4}{|l|}{ Compliance } \\
\hline$\beta$ & 7.7 & 2.5 & 2.1 \\
\hline $95 \% \mathrm{Cl}$ & $(5.9-9.6)$ & $(1.0-3.9)$ & $(0.6-3.6)$ \\
\hline$P$ value & $<.001$ & .001 & .007 \\
\hline \multicolumn{4}{|l|}{ Stiffness index } \\
\hline$\beta$ & -276.3 & -72.7 & -55.1 \\
\hline $95 \% \mathrm{Cl}$ & $(-417.7$ to -135.9$)$ & $(-183.7-38.3)$ & $(-170.6-60.3)$ \\
\hline$P$ value & $<.001$ & .199 & .349 \\
\hline \multicolumn{4}{|c|}{ Pressure-strain elastic modulus } \\
\hline$\beta$ & -0.1 & -0.1 & -0.1 \\
\hline $95 \% \mathrm{Cl}$ & $(-0.2$ to -0.01$)$ & $(-0.2-0.01)$ & $(-0.1-0.03)$ \\
\hline$P$ value & .030 & .070 & .183 \\
\hline
\end{tabular}

${ }^{a}$ Model 1: adjusted for systolic blood pressure mean between visit 2 and visit 5 ( $n=1402$ for WMH and $n=1397$ for total brain volume).

${ }^{\mathrm{b}}$ Model 2: WMH model adjusted for visit 2, patient age, sex, body mass index, HDL cholesterol, current smoking, history of hypertension $(n=1320)$. TBV model adjusted for patient age, sex, black race, fasting glucose, and current smoking $(n=1393)$.

'Model 3: WMH model adjusted for visit 3, patient age, current cigarette smoking, history of hypertension, and voltage of left ventricular leads on ECG $(n=1289)$. TBV model adjusted for patient age, sex, black race, diabetes, and HDL cholesterol level $(n=1296)$

line MR imaging, inability to adjust with more granularity for potential confounders such as medication use or blood pressure, and the absence of data on cerebrovascular stenosis or perfusion during the exposure period.

\section{CONCLUSIONS}

We report a strong association between CS and WMHv on MR imaging performed $>20$ years later and a less consistent association between CS and TBV. A larger WMHv is independently associated with impaired cognitive function, higher risk of both ischemic and hemorrhagic stroke, and worse outcomes after stroke. The Systolic Blood Pressure Intervention Trial-Memory and Cognition in Decreased Hypertension (SPRINT-MIND) trial recently reported that intensive blood pressure control attenuates $\mathrm{WMH}$ progression, ${ }^{15}$ and several other ongoing studies are being planned to evaluate interventions that could reduce WMH. Against this backdrop, our data suggest the importance of CS as an intermediate step in the WMH development pathway, which could be informative for clinical trials of WMH reduction.

Disclosures: Adam de Havenon—RELATED: Grant: American Heart Association, Comments: Research reported in this publication was supported by an American Heart Association Scientist Development Grant 17SDG33670114.* Jennifer J. Majersik—UNRELATED: Grants/Grants Pending: National Institutes of Health/ National Institute of Neurological Disorders and Stroke 5U10NS08660*; Travel/Accommodations/Meeting Expenses Unrelated to Activities Listed: American Academy of Neurology, Comments: Transforming Leaders Program.* Natalia S. Rost-UNRELATED: Consultancy: Sanofi Genzyme, Broadview Ventures, Omniox; Grants/Grants Pending: National Institutes of Health*; Royalties: Up-ToDate. J. Scott McNally — UNRELATED: Grants/Grants Pending: American Heart Association and National Institutes of Health, Comments: amyloid deposition and pre-existing vulnerable carotid plaque features predicting cognitive improvement after surgery, Principal Investigator: J. Scott McNally; National Institutes of Health R01, High-Resolution, Motion-Corrected 3D Cine MRI of carotid plaque, role: Co-l. * ${ }^{*}$ Money paid to institution.

\section{REFERENCES}

1. Au R, Massaro JM, Wolf PA, et al. Association of white matter hyperintensity volume with decreased cognitive functioning: the Framingham Heart Study. Arch Neurol 2006;63:246-50 CrossRef Medline

2. Kuller LH, Longstreth WT Jr, Arnold AM, et al; Cardiovascular Health Study Collaborative Research Group. White matter hyperintensity on cranial magnetic resonance imaging: a predictor of stroke. Stroke 2004;35:1821-25 CrossRef Medline

3. Appelman AP, Exalto LG, van der Graaf $\mathrm{Y}$, et al. White matter lesions and brain atrophy: more than shared risk factors? A systematic review. Cerebrovasc Dis 2009;28:227-42 CrossRef Medline

4. Singer J, Trollor JN, Baune BT, et al. Arterial stiffness, the brain and cognition: a systematic review. Ageing Res Rev 2014;15:16-27 CrossRef Medline

5. Mitchell GF, Guo CY, Benjamin EJ, et al. Cross-sectional correlates of increased aortic stiffness in the community: the Framingham Heart Study. Circulation 2007;115:2628-36 CrossRef Medline

6. van Sloten TT, Protogerou AD, Henry RM, et al. Association between arterial stiffness, cerebral small vessel disease and cognitive impairment: a systematic review and meta-analysis. Neurosci Biobehav Rev 2015;53:121-30 CrossRef Medline

7. Mitchell GF, van Buchem MA, Sigurdsson S, et al. Arterial stiffness, pressure and flow pulsatility and brain structure and function: the Age, Gene/Environment Susceptibility-Reykjavik study. Brain 2011;134:3398-407 CrossRef Medline

8. van Sloten TT, Mitchell GF, Sigurdsson S, et al. Associations be- 
tween arterial stiffness, depressive symptoms and cerebral small vessel disease: cross-sectional findings from the AGESReykjavik Study. J Psychiatry Neurosci 2016;41:162-68 CrossRef Medline

9. Yuan C, Wang J, Ying M. Predictive value of carotid distensibility coefficient for cardiovascular diseases and all-cause mortality: a meta-analysis. PLoS One 2016;11:e0152799 CrossRef Medline

10. Jochemsen HM, Muller M, Bots ML, et al; SMART Study Group. Arterial stiffness and progression of structural brain changes: the SMART-MR study. Neurology 2015;84:448-55 CrossRef Medline

11. Tsao CW, Himali JJ, Beiser AS, et al. Association of arterial stiffness with progression of subclinical brain and cognitive disease. Neurology 2016;86:619-26 CrossRef Medline

12. The Atherosclerosis Risk in Community (ARIC) study: design and objectives-the ARIC investigators. Am J Epidemiol 1989;129:687702 CrossRef Medline

13. Yang EY, Chambless L, Sharrett AR, et al. Carotid arterial wall characteristics are associated with incident ischemic stroke but not coronary heart disease in the Atherosclerosis Risk in Communities (ARIC) study. Stroke 2012;43:103-08 CrossRef Medline

14. Power MC, Lamichhane AP, Liao D, et al. The association of longterm exposure to particulate matter air pollution with brain MRI findings: the ARIC study. Environ Health Perspect 2018;126:027009 CrossRef Medline

15. SPRINT MIND Trial Finds Lower Risk of MCI and Dementia with Lower BP. American College of Cardiology. https://www.acc.org/ latest-in-cardiology/articles/2018/07/26/16/36/sprint-mind-trialfinds-lower-risk-of-mci-and-dementia-with-lower-bp. Accessed June 15,2019 